\title{
Expansion rates of bubble clusters in superheated liquids
}

\author{
Dirk Dietzel ${ }^{1}$, Timon Hitz ${ }^{2}$, Claus-Dieter Munz ${ }^{2}$, Andreas Kronenburg*,1 \\ ${ }^{1}$ Institute of Combustion Technology, University of Stuttgart, Germany \\ ${ }^{2}$ Institute of Aerodynamcis and Gas Dynamics, University of Stuttgart, Germany \\ *Corresponding author: kronenburg@itv.uni-stuttgart.de
}

\begin{abstract}
The present work analyses the growth of multiple bubbles in superheated liquid jets by means of direct numerical simulations (DNS). A discontinuous Galerkin approach is used to solve the Euler equations and an adequate interface resolution is ensured by applying finite-volume sub-cells in cells with interfaces. An approximate Riemann solver has been adapted to account for evaporation and provides consistency of all conserved quantities across the interface. The setup mimics conditions typical for orbital manoeuvring systems when liquid oxygen (LOX) is injected into the combustion chamber prior to ignition. The liquid oxygen will then be in a superheated state, bubble nucleation will occur and the growth of the bubbles will determine the break-up of the liquid jet. The expansion rates of bubble groups under such conditions are not known and standard models rely on single bubble assumptions. This is a first DNS study on bubble-bubble interactions in flash boiling sprays and on the effects of these interactions on the growth rates of the individual bubbles. The present simulations resolve a small section of the jet close to the nozzle exit and the growth of bubble groups inside of the jet is analysed. The results suggest that an individual bubble within the group grows more slowly than conventional models for single bubble growth would predict. The reduction in bubble growth can amount to up to $30 \%$ and depends on the distances between the bubbles and the number of bubbles within the bubble group. In the present case, the volume expansion of the superheated liquid decreases by approximately $50 \%$ if the distance between the bubbles is doubled.
\end{abstract}

\section{Keywords}

Vapour bubble growth, compressible flow, Riemann solver, jet expansion

\section{Introduction}

The attitude control of rockets, space probes and satellites is usually accomplished by utilizing small thrusters. One of the major challenges is the ignition of the mixture of fuel and oxidizer under vacuum conditions. Fuel and oxidizer are injected as liquids and the jet atomization determines the prospects of successful ignition events and of the efficiency of the subsequent combustion process. Due to the low pressure being below saturation conditions, nucleation and growth of the vapour bubbles enhances the disintegration of the liquid jet into a disperse spray. Any current modelling strategies of the so-called flash evaporation process do not resolve bubble nucleation and growth of single bubbles and will require closures for the jet expansion and atomization at sub-grid scale. Models for single bubble growth exist, however, nucleation rates in superheated liquids can be very high and bubbles will interact during their growth process. These interactions are not yet fully understood, but quantifying these small scale processes will be necessary for finding suitable closure models for flash-boiling sprays. One particular aspect are the expansion rates of the bubble groups within the superheated liquid jets that have not been investigated before.

A great effort has been spent on the investigation of flash evaporation, both, experimentally and numerically. The morphology of flashing sprays and the resulting jet angles were investigated experimentally e.g. in [1, 2]. Here, the influence of the ambient temperature and pressure as well as the injection pressure were investigated for different fluids. It was found that, depending on the degree of superheat, spray angles up to $160^{\circ}$ with a homogeneous distribution of fine droplets can be realized. The liquid jets rapidly expand in radial direction immediately after exiting the nozzle. Similar observations were made in various other studies $[3,4,5,6]$. Generally, the experimental results show that models for mechanical break-up are not valid for flash-boiling sprays. Examples for numerical studies on flash evaporation are found in [7, 8, 9, 10, 11]. Different modelling strategies are presented there, ranging from single fluid approaches with additional transport equations for the vapour mass fraction to Euler-Lagrangian simulations, where droplets are explicitly tracked as Lagrangian particles. These are promising approaches for the simulation of the entire spray process, but the initial bubble dynamics in the superheated liquid core cannot be resolved. However, the growth and dynamic behaviour of vapour bubbles in the liquid jet have a significant influence on the jet spreading, break-up and droplet formation. The underlying processes, e.g. the pressure wave dynamics and its effect on the velocity and temperature fields, are not entirely understood and an improved understanding is necessary to obtain further improvements of suitable simulation procedures.

The present work analyses the expansion rates of bubble groups in superheated liquids with the aid of fully resolved direct numerical simulations. The dependency of the growth rates on the bubble number density is investigated for cryogenic oxygen. We use a discontinuous Galerkin (DG) approach to solve for the Euler equations. Special approximate Riemann solvers are applied at the interface between liquid and vapour capturing compressible wave 
dynamics as well as phase change. The interface is accounted for using the Level-set method, and we apply a kinetic relation for determining the evaporation rates. The kinetic relation is calibrated by computations of single vapour bubble growth that correspond to conditions given in the vacuum chamber. A subdivision of a Galerkin cell into finite-volume cells close to the interface allows for a better representation of the interface for a fixed Galerkin resolution. The 3D computations are conducted for one set of initial conditions with a different number of bubble nuclei. The paper is organized as follows: First, the numerical methods for the solution of the conservation equations and the treatment of the phase interface are outlined. Then we give the initial conditions of all physical quantities and detail the calibration of the evaporation model. The geometrical configuration of the three-dimensional simulation is given thereafter. Results of two three-dimenensional DNS are presented and discussed, and the paper is concluded with a summary and outlines future work.

\section{Numerical Methods}

The DNS of multiphase flows with phase change requires a numerical approximation of the conservation equations for mass, momentum and energy. Here, we use a discontinuous Galerkin spectral element method (DGSEM). In contrast to conventional finite-volume approaches, this allows for high order accuracy in the bulk phases without applying computationally expensive numerical schemes. Details of the algorithm are found in [12]. Finite-volume sub-cells are used only in the vicinity of the liquid-vapour interface to improve its representation within the Galerkin cells as described in [13]. To keep track of the interface, we apply the level-set algorithm introduced in [14]. A detailed description and validation of the algorithm consisting of the DGSEM for the compressible Euler equations, including the finite-volume sub-cells and the Level-set algorithm, is given in [15]. The phase change is accounted for by using approximate Riemann solvers along with a kinetic relation for the mass flux estimation [16]. This approximate Riemann solver has been newly developed in [16] and for the first time, it is now applied to problems of single or multiple bubble growth. For coupling the thermodynamics to the hydrodynamics the open-source library CoolProp is used, which is based on a Helmholtz energy functional [17]. In the following sections we give a brief introduction to the different algorithms.

\section{Discontinuous Galerkin spectral element method}

Within the discontinuous Galerkin approach the simulation domain is discretized with a set of control volumes $\Omega$. Each cell can be handled separately and in a first step, a control volume is mapped from physical space, $\mathbf{x}$, to a reference space, $\xi$. The computational cells are spanned to a unit reference element $\mathcal{E}$ of the size $[-1 ; 1]$ in each direction. After multiplying the resulting system of equations by a test function $\phi$, the governing equations read [12]

$$
\int_{\mathcal{E}} J U_{t} \phi \mathrm{d} \boldsymbol{\xi}+\int_{\mathcal{E}} \nabla_{\xi} \cdot \hat{\mathbf{F}}(U) \phi \mathrm{d} \boldsymbol{\xi}=0
$$

with the vector of conservative variables $U$, the flux function $\hat{\mathbf{F}}(U)$ and the Jacobi determinant $J$. An integration by parts of the second term in Eq. (1) gives the weak formulation of the conservation laws,

$$
\frac{\partial}{\partial t} \int_{\mathcal{E}} J U \phi d \boldsymbol{\xi}+\int_{\partial \mathcal{E}}(\hat{\mathbf{F}} \cdot \mathbf{N}) \phi \mathrm{d} S-\int_{\mathcal{E}} \hat{F}(U) \cdot\left(\nabla_{\xi} \phi\right) \mathrm{d} \boldsymbol{\xi}=0 .
$$

The first and the second terms on the left hand side contribute to the time derivative of the conserved quantity and the fluxes across the surface of the DG cell. Each cell additionally provides an inner cell resolution which is accounted for by a continuous in-cell ansatz function. For the numerical integration of the fluxes Lagrange polynomials are used, where the polynomial degree $N$ determines the accuracy of integration. As polynomials are local for one cell only, discontinuities may arise at the cell face and Riemann solvers are used to determine consistent fluxes across the cell boundaries. For the present investigation an HLLC-Riemann solver is applied at single phase DG cell boundaries. As the in-cell polynomials are continuous, the interface between liquid and vapour cannot be accounted for and a DG cell is sub-divided into finite-volume cells in the vicinity of the interface [13]. The time integration is accomplished by a third order Runge-Kutta method.

\section{Interface treatment and phase change modeling}

The interface between gas and liquid is tracked using the level-set approach introduced by Sussman et al. [14]. The signed distance function $\Phi(\mathbf{x}, t)$ is transported as

$$
\frac{\partial \Phi}{\partial t}+\mathbf{u}_{\llcorner S} \cdot(\nabla \Phi)=0
$$

The transport velocity of the level-set, $\mathbf{u}_{\mathrm{LS}}$, accounts for the movement of the zero-isosurface of $\Phi$. In the interface cells $\mathbf{u}_{\mathrm{LS}}$ is obtained from the liquid-vapour jump conditions for the mass and the momentum balance. This velocity is then extended to several neighbouring cells. The level-set determines the distance to the interface at every position in the flow field and the zero isocontour of $\Phi$ defines the position of the interface. Since the transport further away from the interface must not necessarily account for the displacement of the nearest interface, the Hamilton-Jacobi 


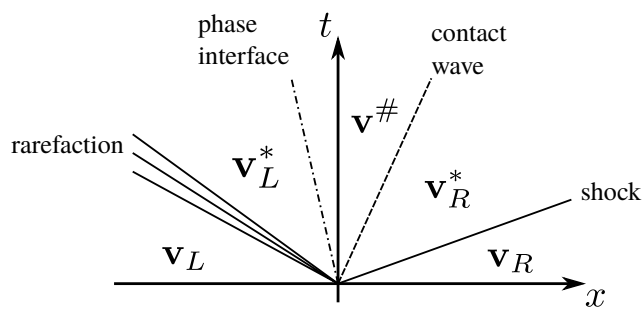

Figure 1. Riemann wave fan of a two-phase Riemann problem including phase change. State vector $\mathbf{v}=(\rho, \rho u, \rho e, p)^{t}$. Here, the different state vectors describe the initial liquid state $\left(\mathbf{v}_{L}\right)$, rarefied liquid $\left(\mathbf{v}_{L}^{*}\right)$, evaporated fluid $\left(\mathbf{v}^{\#}\right)$, compressed vapour $\left(\mathbf{v}_{R}^{*}\right)$ and the initial vapour state $\left(\mathbf{v}_{R}\right)$.

equation

$$
\frac{\partial \Phi}{\partial \tau}=S_{\epsilon}\left(\Phi_{\tau=0}\right)(|\nabla \cdot \Phi|-1)
$$

is iterated for a pseudo time $\tau$. When Eq. (4) has converged to a steady state the distance property of $\Phi$ is recovered fulfilling the condition $|\nabla \cdot \Phi|=1$.

At the phase interface between liquid and vapour, a strong contact discontinuity usually appears, particularly for density and pressure, and fluxes across the interface cannot be determined in the same manner as in the bulk phases. In addition, phase change shall be considered within the present investigation which complicates the Riemann problem at the phase interface. In order to guarantee thermo- and hydrodynamically consistent fluxes across the phase interface including the modeling of phase transition, special approximate Riemann solvers are applied as introduced in [16]. Figure 1 depicts a typical two-phase Riemann problem including rarefaction, shock and contact waves as well as the phase interface as an additional discontinuity. The different fluid states describe the initial liquid state, rarefied liquid, evaporated fluid, compressed vapour and the initial vapour state from the left to the right, respectively. An exact solution of the Riemann problem requires an iterative solution of the RankineHugoniot jump conditions across the different waves. Within the rarefaction fan, an isentropic flow is assumed, and the phase interface is modeled as under-compressive shock wave. Given a suitable expression that determines the evaporating mass, the solution of the resulting system of equations provides physically exact interface fluxes but its iterative solution procedure is computationally expensive. Thus, an approximate Riemann solver is introduced, where the complexity of the mathematical problem is reduced. The simplifications include an estimation of the outer wave speeds (rarefaction and shock waves) as well as omission of the contact wave. The iteration procedure now simplifies to finding the interfacial mass flux where we use the kinetic relation [18]

$$
\frac{c_{\text {ent }} \dot{m}^{\prime \prime 2}}{T_{\text {ref }}}=\llbracket \rho\left(u-a_{\text {if }}\right)\left(s-s_{\text {sat }}\left(T_{\text {ref }}\right)\right) \rrbracket .
$$

Here, the square brackets indicate the jump across the interface, i.e. the difference between liquid and vapour state. The mass flux $\dot{m}^{\prime \prime}$ is estimated comparing the deviation of the local entropy, $s$, to the saturation entropy, $s$ sat, between the liquid and the vapour phases. The coefficient $c_{\text {ent }}$ guarantees a consistent scaling of the entropy production with mass flux. Details of the Riemann solver are found in [16]. The calibration of $c_{\text {ent }}$ is presented in the next section. As continuous polynomials are used within one DG cell, it is not possible to capture the interface discontinuity between polynomial nodes, and the liquid-vapour interface must lie on DG cell interfaces. To enhance the interface resolution, the polynomial nodes are treated as finite volume (FV) cells in the vicinity of the interface by subdividing each DG cell into $N+1$ FV sub-cells per spatial direction [13]. These cells are decoupled from their polynomial ansatz function and discontinuities may arise between them. For the present investigation a sharp interface approach is used. Every computational cell is considered as either liquid or gaseous and there are no mixed cells. Thus, interfacial fluxes can be determined directly across FV cell faces in the refined region and the interface is shifted from one cell face to the next whenever the sign of $\Phi$ changes. It has to be noted that this approach is not strictly conservative but is needed to avoid a smearing of the interface over several computational cells.

\section{Setup}

\section{Physical initial conditions}

The physical initial conditions are given in Tab. 1. We have chosen an ambient temperature of $T=120 \mathrm{~K}$ and an ambient pressure of $p_{\infty}=2.0 \cdot 10^{5} \mathrm{~Pa}$. The conditions inside of the bubble are typically estimated assuming saturation conditions at the ambient temperature with $p_{\text {bub }}=p_{\text {sat }}(T)$. The initial bubble radius results from the Young-Laplace law as [19]

$$
r_{0}=\frac{2 \sigma}{p_{\text {sat }}-p_{\infty}},
$$


Table 1. Initial conditions for liquid oxygen. The initial velocity is zero everywhere.

\begin{tabular}{c|ccc}
\hline & vapour bubble & liquid & ambient vapour \\
\hline$\rho\left[\mathrm{kg} / \mathrm{m}^{3}\right]$ & 39.308 & 969.208 & 6.752 \\
$p[\mathrm{~Pa}]$ & $1.022 \cdot 10^{6}$ & $2.045 \cdot 10^{5}$ & $2.045 \cdot 10^{5}$ \\
$T[\mathrm{~K}]$ & 120 & 120 & 120
\end{tabular}
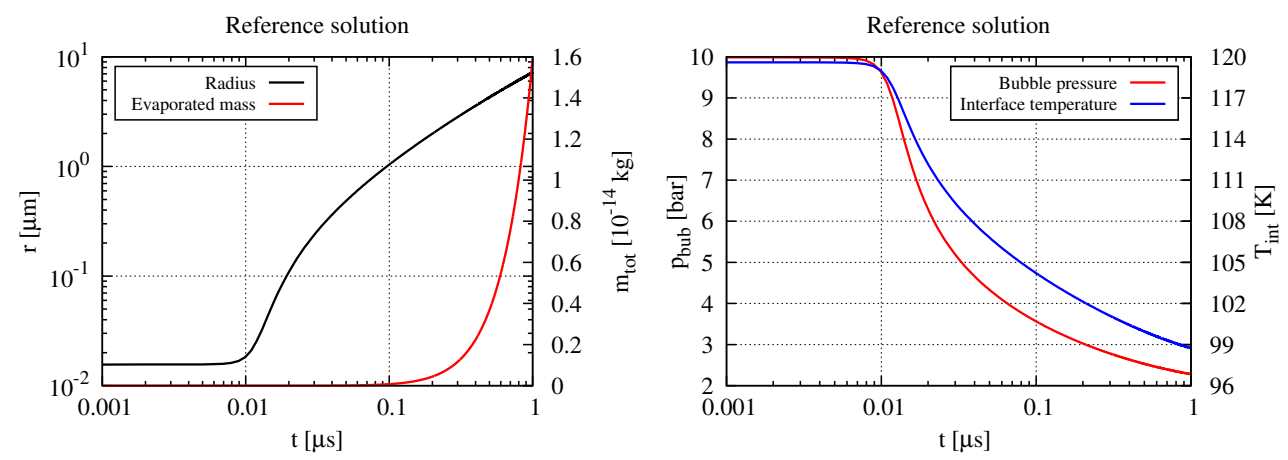

Figure 2. Left: Time evolution of the bubble radius (black line) and the accumulated mass (red line). Right: Time evolution of the bubble pressure (red line) and the interface temperature (blue line). Results are obtained from the solution of the Rayleigh-Plesset equation coupled to the temperature equation in the liquid.

where $\sigma$ is the surface tension coefficient. It is $\sigma=0.006 \mathrm{~N} / \mathrm{m}$ for liquid oxygen at $T=120 \mathrm{~K}$ giving $r_{0}=0.015 \mu \mathrm{m}$. The saturation pressure inside the bubble and the ambient pressure give a pressure ratio of $R_{p}=p_{\text {sat }} / p_{\infty}=5$. Note that flash-boiling with liquid jets containing numerous bubbles occurs typically at much higher pressure ratios (e.g. [2]). However, the growth of single vapour bubbles is found to be relatively insensitive to $R_{p}$ for the time range considered and in the present investigation, the bubble number density does not depend on the choice of $R_{p}$.

\section{Scaling of the evaporation model}

As a reference case for calibrating the entropy production coefficient, $c_{\text {ent }}$ (see Eq. (5)), the growth of a single bubble is used. Figure 2 depicts the reference solution obtained by solving the Rayleigh-Plesset equation (see [20]) including a coupling with the temperature equation in the liquid as given in [21]. The black line in Fig. 2 (left) shows the bubble radius over time, and it is seen that it has grown to approximately $r=10 \mu \mathrm{m}$ after $t=1 \mu \mathrm{s}$. This radius corresponds to the average bubble size when bubbles coalesce for conditions given in our first reference case (see below). The red line in the left sub-figure in Fig. 2 shows the accumulated evaporated mass over time. Only at time scales of the order of a micro-second, we observe a significant rise in the evaporated mass. This indicates that bubble expansion at time scales one or several orders smaller than the final time (here one micro-second) will not affect the volume expansion of the liquid jet and the dynamics related to the bubble expansion at these scales do not need to be considered. In Fig. 2 (right), the pressure inside of the bubble (red line) and the interface temperature (blue line) are depicted. We observe a fast decrease in the interface temperature due to the latent heat of vaporization. As the bulk liquid remains at a high temperature, a thermal boundary layer develops. The pressure inside of the bubble decreases with the interface temperature and it quickly reaches pressures close to the ambient pressure after $1 \mu \mathrm{s}$. Typically, bubble growth is divided in an early inertia driven stage and a subsequent stage driven by heat diffusion (see e.g. [19]). However, the decreased pressure seen in Fig. 2 starts to affect the bubble growth even at early times. Thus, despite an expectancy of a slow heat diffusion process, it needs to be included at the small time scales if the effects of evaporative cooling and pressure drop are to be considered. In order to account for this effect the evaporation model in Eq. (5) is scaled to match the total evaporated mass after $t=1 \mu \mathrm{s}$. In Fig. 3 the solution from the DG solver with $c_{\mathrm{ent}}=540$ is compared to the reference solution. The initial radius in the DG solution is increased to $r_{0, D G}=1.0 \mu \mathrm{m}$. This corresponds to the radius at $t=0.1 \mu \mathrm{s}$ for the reference solution, where significant mass accumulation starts. The increase in the initial radius allows for a coarser mesh resolution and thus, a coarser time step such that the computational effort for the three-dimensional computations stays acceptable. The left subfigure of Fig. 3 shows the bubble surface over time. Differences are only observed at early times, when the difference in the initial radii is not compensated. The middle and the right sub-figures of Fig. 3 depict the volume change of the bubble and the total evaporated mass, respectively. The results from the DG solver match very well with the reference solution at all times, and the difference in initial bubble radius should therefore not unduly affect the dynamics of jet expansion and breakup. Note, that the calibration is specific for the present ambient conditions and needs to be adapted accordingly if conditions change. 

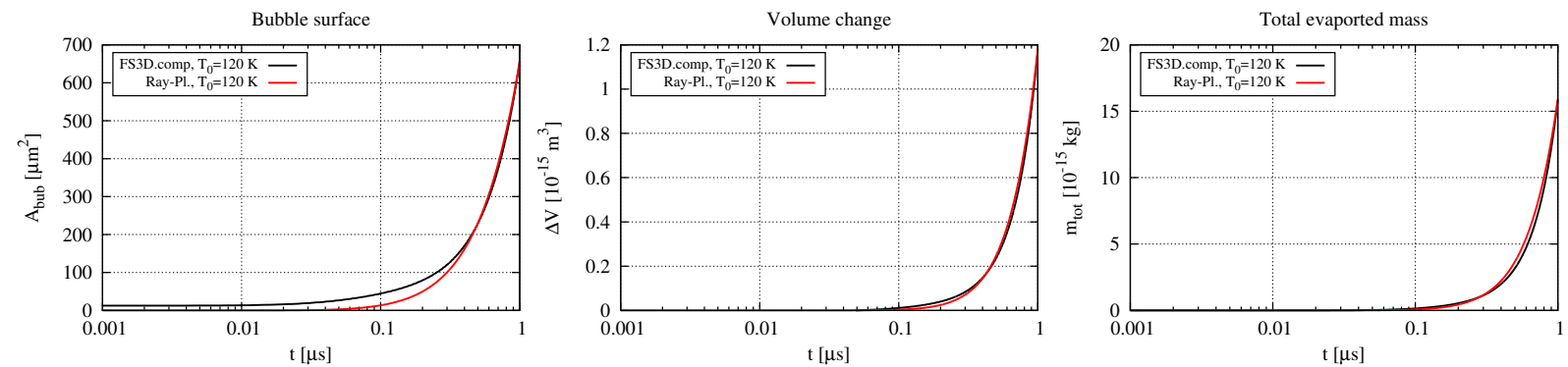

Figure 3. Bubble surface (left), volume change (middle) and total evaporated mass (right) of the scaled computations for single bubble growth. The red and the black lines represent the reference solution and the solution obtained from the DG solver, respectively.
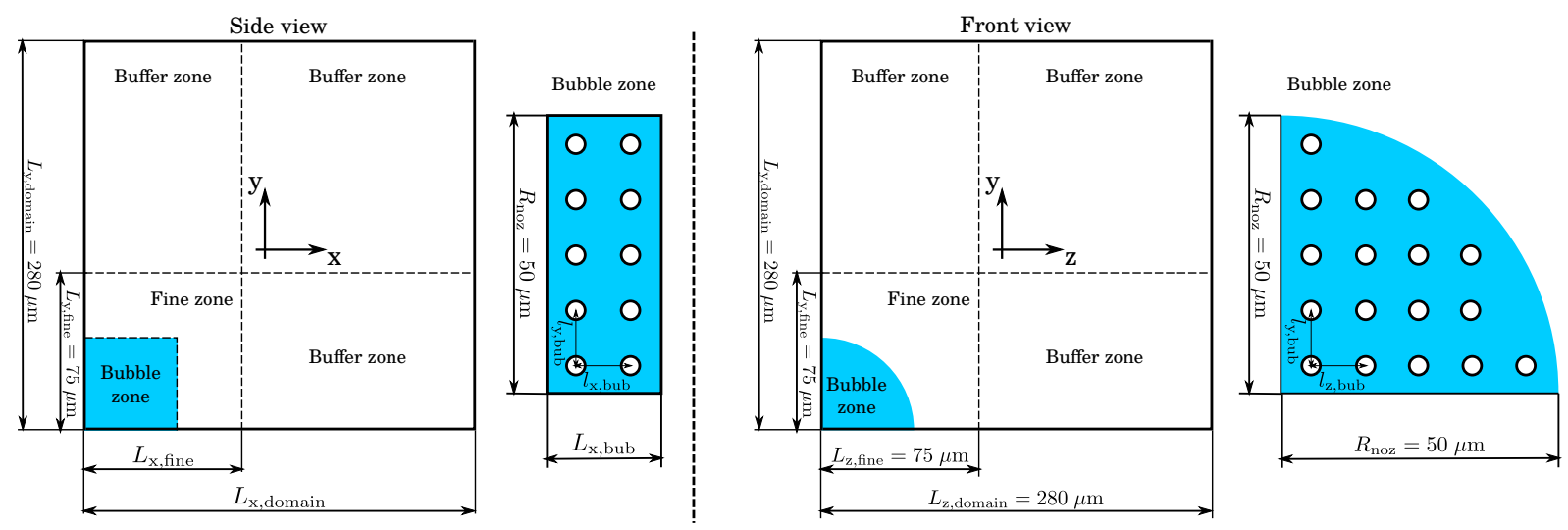

Figure 4. Geometrical setup of the three-dimensional simulations. The bubble zone represents the tip of a liquid jet immediately after the nozzle exit.

\section{Geometry and Numerics}

In the present investigation we focus on the small scale processes close to the nozzle exit, where the growth of vapour bubbles begins to affect the dynamics of the liquid jet. The computational domain is depicted in Fig. 4. A small liquid core is considered where a fixed number of bubbles is prescribed (bubble zone). The lengths of the bubble zone in $y$ - and $z$-directions are defined by the nozzle radius. Typical nozzle radii in corresponding experimental investigation of the German national aeronautics and space research centre (DLR Lampoldshausen) lie in between $R_{\mathrm{noz}}=50 \mu \mathrm{m}$ and $R_{\mathrm{noz}}=500 \mu \mathrm{m}$ and we choose $R_{\mathrm{noz}}=50 \mu \mathrm{m}$ for the present case. The fine zone in Fig. 4 has the same equidistant mesh resolution as the bubble zone, and therefore guarantees a sufficient resolution during liquid core expansion. The grid spacing in the fine zone is chosen such that a bubble diameter is initially resolved with eight FV-cells. In the buffer zone the cells are stretched, and this zone is needed to reduce the influence of the outflow boundary conditions applied in the positive $x-, y-$ and $z$-directions. At all other boundaries symmetry conditions are applied imposing some degree of rotational symmetry of the expansion process. The mesh dimensions in $y$ - and $z$-directions are the same for all simulations and they are depicted in Fig. 4 . We set up two cases with a different bubble spacing. The cases are labelled Case 1 and Case 2. The number of bubbles in the $y$ - and $z$-direction is $N_{\text {bub,y }}=N_{\text {bub,z }}=5$ for Case 1 and $N_{\text {bub,y }}=N_{\text {bub,z }}=3$ for Case 2 . The distance of the bubbles is $l_{\mathrm{bub}}=10 \mu \mathrm{m}$ and $l_{\mathrm{bub}}=20 \mu \mathrm{m}$, respectively, and equal in all three spatial directions. The number of bubbles in $x$-direction is the same in Case 1 and 2 but the length of the bubble zone is extended such that we have a consistent spacing of the bubbles according to the other directions. In that way we resolve two bubble layers in the $x$-direction in both cases allowing for a comparison between bubbles located in the center and at the edges of the bubble group. The total number of bubbles resolved is 34 in Case 1 and twelve in Case 2. The axial extension of the initial liquid core corresponds to the estimated penetration of the liquid into the vacuum chamber during the time needed for jet break-up. All the geometric data are given in Tab. 2.

\section{Results and discussion}

Three-dimensional results are shown in Fig. 5 for Case 1. The curved iso-surfaces indicate the bubbles and the liquid jet, respectively. The $x-$ and the $z$-normal plane show the velocity fields. The inner bubbles grow isotropically while the outer bubbles expand stronger towards the jet surface (e.g. top right bubbles in Fig. 5). In the direction of the jet surface, bubbles are permanently facing the low-pressure environment being the driving force of bubble expansion. This is different in the jet center, where the expansion of the bubbles is more sensitive to the local pressure fluctuations and the resistance effectuated by the neighbouring bubbles decreases the expansion rates. 
Table 2. Geometrical specifications for Case 1 and Case 2.

\begin{tabular}{c|cc}
\hline & Case 1 & Case 2 \\
\hline$N_{\text {bub }, \mathrm{x}}$ & 2 & 2 \\
$N_{\text {bub,yz }}$ & 5 & 3 \\
$l_{\text {bub }}[\mu \mathrm{m}]$ & 10 & 20 \\
$L_{\mathrm{x}, \text { bub }}[\mu \mathrm{m}]$ & 20 & 30 \\
$L_{\mathrm{x}, \text { fine }}[\mu \mathrm{m}]$ & 30 & 45 \\
$L_{\mathrm{x}, \text { domain }}[\mu \mathrm{m}]$ & 235 & 250
\end{tabular}

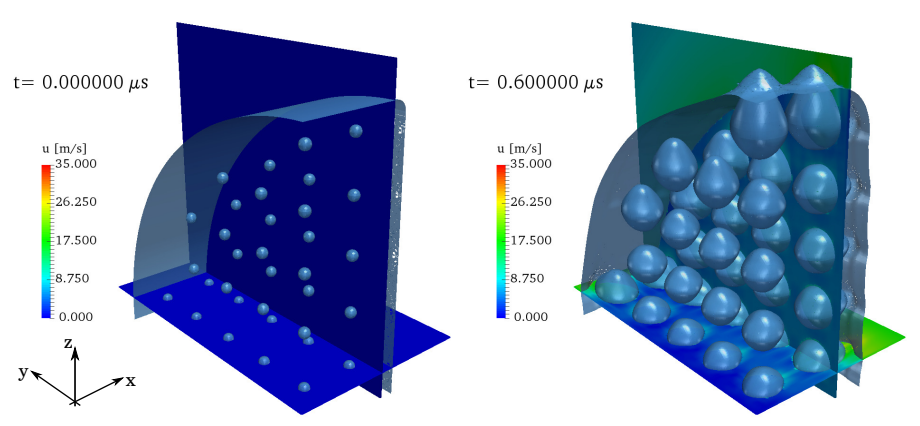

Figure 5. Expansion of vapour bubbles in a liquid jet. The curved iso-surfaces represent the bubble and jet surfaces, while the velocity field is shown in the $\mathrm{x}$ - and $\mathrm{z}$-normal slices. Left: Initial conditions. Right: Solution after $t=0.6 \mu \mathrm{s}$.

Furthermore, we observe an expansion of the liquid jet despite the rather short time interval $(t=0.6 \mu \mathrm{s})$. Twodimensional plots of density, velocity and pressure fields are depicted in Fig. 6 . The initial conditions in the top row are compared to the solution after $t=0.6 \mu \mathrm{s}$ in the bottom row. The density fields in the left column qualitatively confirm the impression from the three-dimensional plots. The outer bubbles grow faster and become non-spherical while inner bubbles expand isotropically at a lower rate. All inner bubbles grow approximately at the same rate. The velocity field is depicted in the middle column of Fig. 6 . The expansion velocity of $u \approx 15 \mathrm{~m} / \mathrm{s}$ is roughly of the order of typical jet velocities for the corresponding DLR experiments. Thus, the bubble expansion can have a considerable influence on the jet break-up. Peak velocities of up to $u=30 \mathrm{~m} / \mathrm{s}$ at the jet surface arise locally where the bubbles grow at the highest rate and where deformation of the jet surface is observed and jet break-up imminent. Note that the high velocities in the surrounding vapour phase do not only result from jet expansion but also from the evaporation at the jet surface. It is also seen that the velocities decrease rapidly towards the jet center. This may change when the outer bubbles merge and the jet surface breaks up by forming droplets, which will be investigated in future studies. In the right column of Fig. 6 the pressure fields are depicted. The pressure in the center is slightly higher than in the environment and at the jet surface. The evaporation rate in Eq. (5) is determined by the deviation of the local entropy from the entropy at saturation conditions. The increase in pressure decreases the degree of superheat in the liquid and the difference of the local entropy to the saturation entropy in Eq. (5) is reduced resulting in lower evaporation rates. In addition, the mechanical resistance against the bubble expansion is higher for higher liquid pressures. The pressure increase originates from pressure fluctuations travelling continuously between the jet center and the jet surface. The temporal average of the local pressures determines the deviations of the growth rates between the inner and outer bubbles. Here, the growth rate is defined as the time derivative of the bubble radius $\mathrm{d} r_{\mathrm{bub}} / \mathrm{d} t$, i.e. it corresponds to the gradient of the functions plotted in the $r-t$-diagrams. The density, velocity and pressure fields of Case 2 are similar (not shown here).

Figures 5 and 6 reveal a variation of growth rates dependent on the position of the bubble within the bubble array. It is apparent that single bubble growth cannot capture the average growth process accurately and a quantification of characteristic growth rates is required. For the present case the growth rates of all inner bubbles and all outer bubbles are averaged. Here, we use the bubble volume and calculate an equivalent radius of the corresponding spherical bubble. All bubbles directly facing the jet surface to the positive $x-, y-$ or $z$-direction are marked as outer bubbles, while the bubbles that only face neighbouring bubbles are inner bubbles. The left subfigure in Fig. 7 depicts the radii over time for the outer and inner bubbles for Case 1. The solution for the single bubble growth is given as reference and the growth rate for the bubble located at the lowest $x-, y$ - and $z$-coordinate is also plotted as it was expected to present a limiting case. It seems, however, that the exact position in the array is not so important. The innermost bubble grows approximately at the same rate as the average of the inner bubbles as indicated in the figure by the red and black solid lines, respectively. This is consistent with the qualitative impression from the twoand three-dimensional plots. The growth rate of the inner bubbles is approximately $30 \%$ lower than the growth rate of a single bubble (dash-dot line). A jet expansion based on growth rates of single bubbles would then be prone to model inaccuracies. The outer bubbles (dashed lines in Fig. 7) grow faster than the inner bubbles by approximately $20 \%$ but the expansion is still lower than for single bubbles. As a result, the outer bubbles will coalesce first and the subsequent jet disintegration will propagate from the outside towards the jet center. The right subfigure shows 

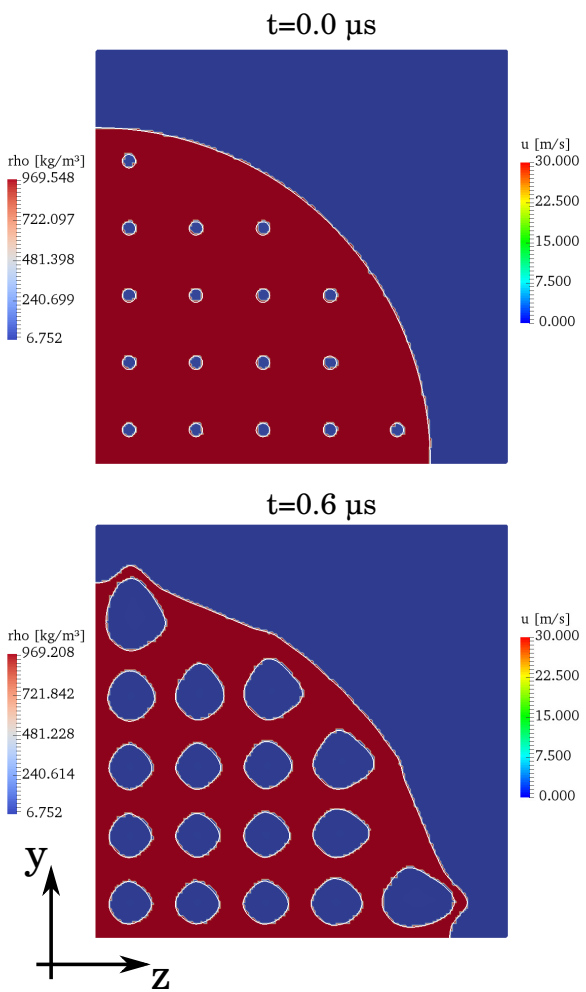
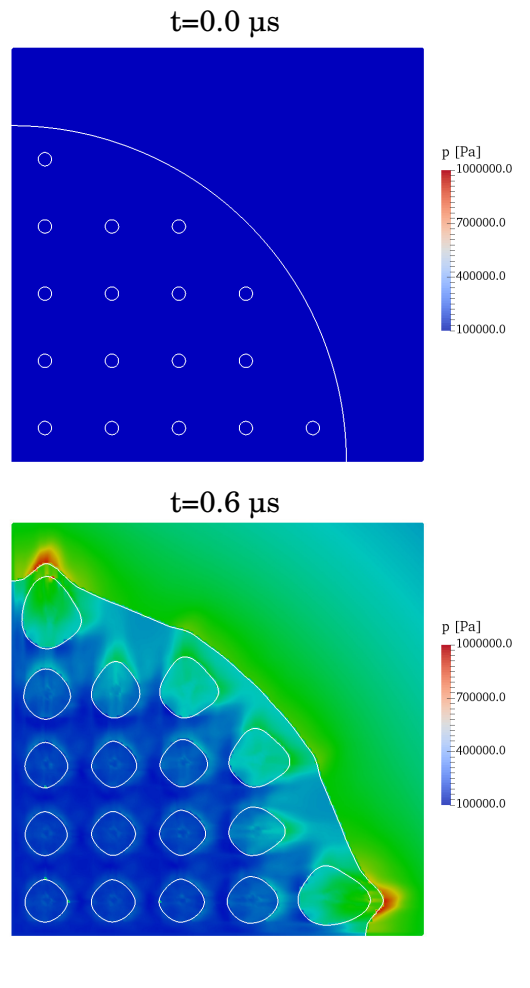

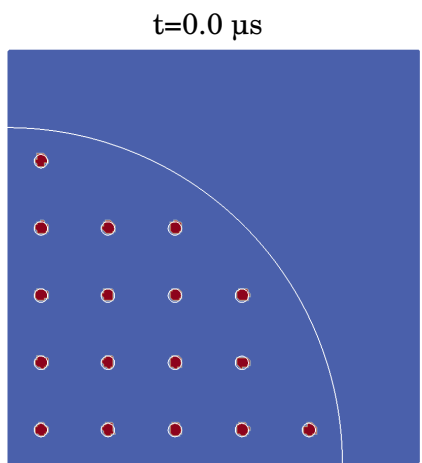

$\mathrm{t}=0.6 \mu \mathrm{s}$

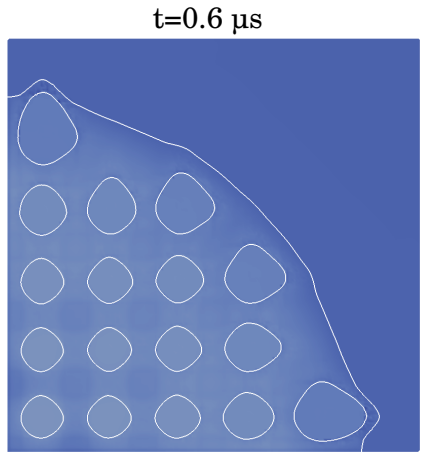

Figure 6. Density (left), velocity (middle) and pressure (right) fields in an $y-z-$ plane. Top row: Initial conditons. Bottom row: Solution after $t=0.6 \mu \mathrm{s}$.

results of Case 2. Qualitatively, there is little difference between the two cases. However, the absolute growth rates of Case 1 and Case 2 deviate. Both, the inner and the outer bubbles grow faster by $15 \%$ in Case 2 compared to Case 1. For the present test condition, we illustrate the dependency of individual bubble growth rates on the bubble density (i.e. the number of bubbles per volume) and their position within the liquid jet. The expansion of the liquid jet will be determined by the total expansion of all bubbles. The bubbles grow faster for Case 2, but less bubbles are contained in the liquid and the total expansion of the jet is smaller. Table 3 shows the volume of the jet as the sum of the liquid and the bubble volume. The volume change compared to the initial solution is $20 \%$ in Case 1 and $7 \%$ in Case 2. The absolute change in the jet volume in Case 2 is $54 \%$ of the absolute change in Case 1 (consult the fourth column in Tab. 3). This demonstrates the sensitivity of the jet expansion to the bubble growth and its dependence on both, the local growth rates and the bubble density. The volume change based on the solution of the growth model for a single bubble multiplied with the number of bubbles in the liquid jet would be doubled in Case 1 and would be overpredicted by $25 \%$ in Case 2 . Yet, the parameter range in our investigation is small and a more comprehensive study for wide parameter ranges and at simulation times including droplet formation will be needed to derive models for flash-boiling that are valid for a wider range of conditions with varying nucleation rates.

Table 3. Volume expansion of the liquid jet for Case 1 and Case 2.

\begin{tabular}{c|cccc}
\hline & $V(t=0.0 \mu \mathrm{s})\left[\mathrm{m}^{3}\right]$ & $V(t=0.6 \mu \mathrm{s})\left[\mathrm{m}^{3}\right]$ & $\Delta V / \Delta V_{\text {Case } 1}$ & $\Delta V[\%]$ \\
\hline Case 1 & $3.927 \cdot 10^{-14}$ & $4.705 \cdot 10^{-14}$ & 1 & 20 \\
Case 2 & $5.890 \cdot 10^{-14}$ & $6.307 \cdot 10^{-14}$ & 0.54 & 7
\end{tabular}

\section{Conclusions}

In the present paper the expansion rates of multiple bubbles in a superheated liquid jet are investigated. The numerical simulations are conducted using a discontinuous Galerkin solver. The fully compressible approach requires a modified approximate Riemann solver that accounts for phase change and captures pressure wave dynamics. The evaporation model requires closure which is realized by the calibration of DG calculations with the RayleighPlesset equation for cases of single bubble growth. We show that the dynamics of early bubble growth becomes unimportant at larger time scales, and the total volume expansion obtained with the DG solver matches well with the reference solution throughout the entire simulation time.

The calibrated model is used to investigate the growth rates in a three-dimensional liquid jet containing multiple 

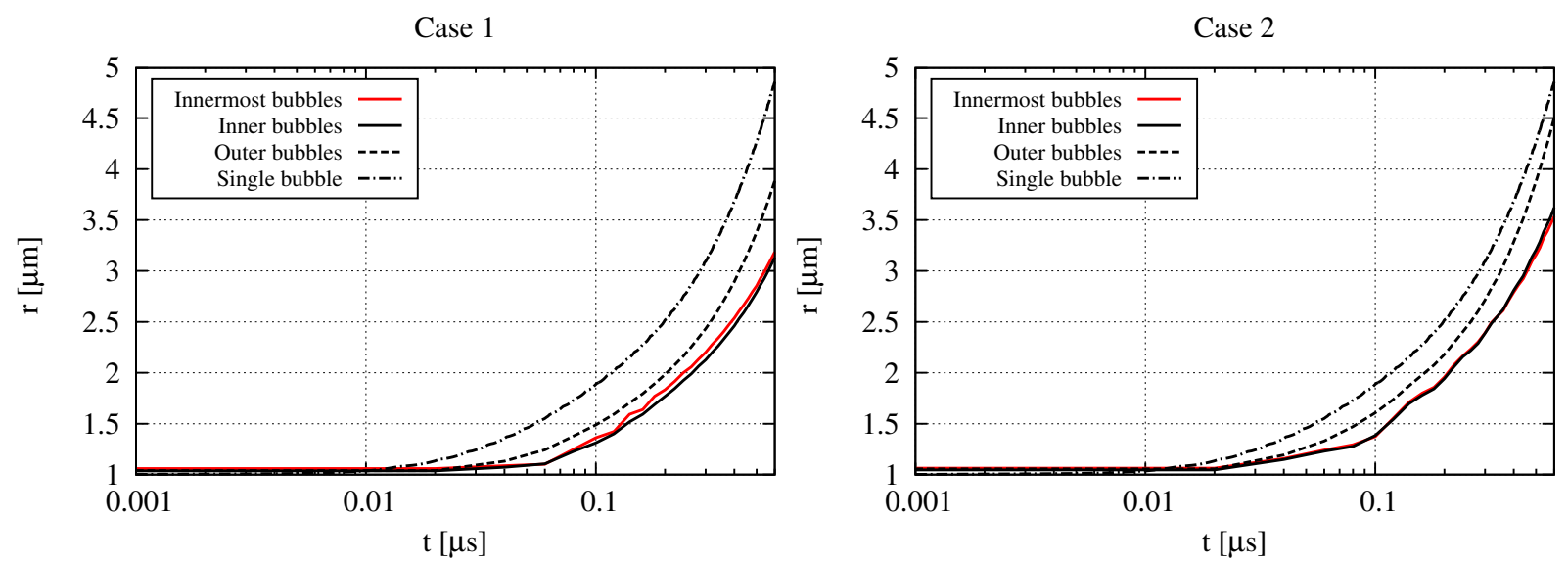

Figure 7. Comparison of bubble growth rates of inner bubbles, outer bubbles and single bubble growth. Left: Case 1. Right: Case 2:

bubbles. We observe a strong growth of the bubbles resulting in high expansion velocities for the liquid jet in the axial and radial direction. The velocities are of the order of the characteristic mean flow velocities and they are reached at very short time scales. Moreover, we observed non-homogeneous growth of the bubbles and have quantified the deviation to single bubble growth. It is found that bubbles in the center expand more slowly than bubbles at the edges of the jet, however, even bubbles right at the jet interface do not reach expansion rates as were predicted by existing expressions for single bubble growth. The present investigation is a first approximation and will be extended to include a wider parameter range. Particularly, the influence of the bubble number density has to be investigated and computations that capture the entire jet break-up due to bubble growth will be attempted. The bubble number density is a function of the ambient temperature and the pressure ratio $R_{p}$. It may not be possible to achieve realistic bubble number densities for high $R_{p}$, however, the current studies show that there is little influence of the exact position within the array on bubble growth rates (as long as the bubble does not face the jet interface). This may allow for future computations of a reduced domain size located in the immediate vicinity of the jet surface where much higher nucleation rates could be realized.

\section{Acknowledgements}

The authors acknowledge the financial support by the Deutsche Forschungsgemeinschaft (DFG) as part of the Collaborative Research Center SFB TRR 75 "Droplet dynamics under extreme ambient conditions" held by University of Stuttgart and University of Technology Darmstadt.

\section{References}

[1] Lamanna, G. et al., 2014, International Journal of Multiphase Flow, 58, pp. 168-184.

[2] Lamanna, G. et al., 2015, Atomization and Sprays, 25 (10), pp. 837-856.

[3] Günther, A. and Wirth, K.E., 2013, International Journal of Heat and Mass Transfer, 64, pp. 952-965.

[4] Huang, Y., et al., 2016, Energy Conversion and Management, 108, pp. 68-77.

[5] Ju, D. et al., 2015, International Journal of Multiphase Flow, 75, pp. 224-236.

[6] Thompson, A.S. and Heister, S.D., 2016, Atomization and Sprays, 26 (7), pp. 633-658.

[7] Neroorkar, K. and Schmidt, D., 2011, Fuel, 90, pp. 665-673.

[8] Calay, R.K. and Holdo, A.E., 2008, Journal of Hazardous Materials, 154, pp. 1198-1209.

[9] Downar-Zapolski, P. et al., 1996, International Journal of Multiphase Flow, 22 (3), pp. 473-483.

[10] Zeng, Y. and Lee, C.-F.F., 2001, Combustion, Science and Technology, 169, pp. 45-67.

[11] Saha, K. et al., 2016, Journal of Energy Resources Technology, 138 (052208), pp. 1-11.

[12] Hindenlang, F. et al., 2012, Computers \& Fluids, 61, pp. 86-93.

[13] Fechter, S. et al., 2012, In: 17th International Conference in on Computational Fluid Dyanmics, Big Island, Hawaii, 9-13 July 2012.

[14] Sussman M. et al., 1994, Journal of Computational Physics, 114, pp. 146-159.

[15] Fechter S. et al., 2015, International Journal in Numerical Methods in Fluids, 78 (7), pp. 413-435.

[16] Fechter S., 2015, Compressible Multiphase Simulation using a discontinuous Galerkin scheme, PhD. thesis, Universität Stuttgart.

[17] Bell, H. et al., 2014, Industrial \& Engineering Chemistry Research, 53, pp. 2498-2508.

[18] Fechter S. et al., 2017, Journal of Computational Physics, 336, pp. 347-374.

[19] Brennen, C. E., 1995, "Cavitation and bubble dynamics". Oxford University Press.

[20] Plesset, M.S. and Prosperetti, A., 1977, Annual Review of Fluid Mechanics, 9, pp. 145-185.

[21] Lee, M. and Merte, H. Jr., 1996, International Journal of Heat and Mass Transfer, 39 (12), pp. 2427-2447. 